\title{
Evaluation and comparison of a machine learning cloud identification algorithm for the SLSTR in polar regions
}

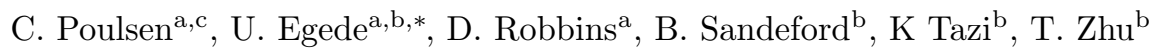 \\ ${ }^{a}$ Monash University, Melbourne, Australia \\ ${ }^{b}$ Imperial College London, London, United Kingdom \\ ${ }^{c} R A L$ Space, STFC, United Kingdom
}

\begin{abstract}
A Feed Forward Neural Net (NN) approach to distinguish between clouds and the surface has been applied to the Sea and Land Surface Temperature Radiometer in polar regions. The masking algorithm covers the Arctic, Antarctic and regions typically classified as the cryosphere such as northern hemisphere permafrost. The mask has been trained using collocations with the CALIOP active lidar, which in narrow strips provide more accurate detection of cloud, and was subsequently evaluated as a function of cloud type and surface type. The mask was compared with the existing operational Bayesian and Empirical cloud masks by eye and also statistically using CALIOP data. It was found to perform exceptionally well in the polar regions. The Kuiper skill score improved from 0.28, for the operational Bayesian and 0.17 for the Empirical masks to 0.77 for the NN. The NN algorithm also has a much more homogeneous performance over all surface types. The key improvement came from better identification of clear scenes; for the NN mask, the same performance in terms of contamination of cloudy pixels in the sample of identified clear pixels can be achieved while retaining $40 \%$ of the clear pixels compared with $10 \%$ for the operational cloud identification. The algorithm performed with almost the same skill over sea and land. The best performance was achieved for opaque clouds while transparent and broken clouds showed slightly reduced accuracy.
\end{abstract}

Keywords: SLSTR, Sentinel-3, Cloud mask, poles, Neural net

\footnotetext{
${ }^{*}$ Corresponding author

Email address: ulrik.egede@monash.edu (U. Egede)
}

Preprint submitted to Remote Sensing of Environment

July 10, 2020 


\section{Introduction}

Clouds play an important role in moderating the solar radiation incident on Earth and regulating the amount of radiation back to space. The balance of radiation reflected and emitted depends critically on the coverage, temperature 5 and albedo of the cloud. How these cloud properties will change in a warming climate is still highly uncertain as outlined in the latest IPCC report, Stocker et al. (2013).

The polar regions are particularly important moderators of the Earth global radiation balance directly and indirectly through circulation changes and global teleconnections. Over the course of the year, heat moves away from the equator into the polar regions and escapes through the atmosphere; the polar region typically gives off more heat than it absorbs.

Changes to the global climate can be amplified over the polar regions. There is evidence that the polar regions are changing faster than other regions. In the IPCC Special Report on the Ocean and Cryosphere in a Changing Climate, Pörtner et al. (2019), the Arctic sea ice extent was shown to have decreased significantly in the past few decades. In the Antarctic, the changes are more uncertain with the sea ice extent increasing until 2014 and then more recently decreasing at rates higher than those observed in the Arctic, described in Parkinson (2019). The impact of clouds on the radiative forcing in the polar regions is complex and can result in both positive or negative feedbacks depending on how the amount and type of clouds changes in response to global warming and sea ice loss. This has been outlined in Goosse et al. (2018) and Huang et al. (2019).

25 The permafrost region as defined in Obu et al. (2019), is a large carbon store sensitive to climate changes with effects of thawing permafrost on the formation of clouds in the region and any subsequent feedback effect in this region is also currently uncertain. Further studies of the correlations between clouds and effects that have a large impact of the climate can be improved by having a better performing cloud mask that performs uniformly across all surface types. 
Cloud masking is essential in its own right for monitoring of trends in cloud coverage and properties. In addition, it is an important first step in developing satellite retrieval algorithms of surface properties or atmospheric variables. Whether the aim is to observe the surface temperature, water vapour or aerosols, cloud masking over the polar regions is particularly challenging as frozen ground, sea ice and snow have similar spectral properties to clouds i.e. the surface is white and bright and the surface temperature is cold. For these reasons, cloud masking algorithms that work well over land and sea surfaces outside polar regions, typically perform poorly within them. As a consequence of this, in order to reduce cloud contamination biases in surface variable retrievals, existing algorithms typically over mask in these regions, and hence the number of pixels in an image that are classified as not having cloud cover is dramatically reduced compared to what is possible with a better performing algorithm.

There is presently a paucity of ground-based observations in the polar re45 gions and in the past the situation was even worse. Satellite observations are thus important and can be used to fill the spatial and temporal gaps necessary to monitor the changes in the polar regions. When considered together, the observations may provide insight into exchanges in radiation between the surface, ocean and atmosphere that could have impacts for atmospheric and oceanic circulation. Satellite observations can be used to evaluate the representation of these effects in climate models.

In this paper we present a cloud identification algorithm developed for the SLSTR (Sea and Land Surface Temperature Radiometer) instrument on board both Sentinel-3A and Sentinel-3B. The algorithm has been developed specifically for the polar regions encompassing ocean, sea ice, inland sea, land and permafrost.

The cloud identification algorithm uses a Feed Forward Neural Net (NN) algorithm that has been trained with collocated data from the CALIOP Instrument. The algorithm is presented as well as a sensitivity study and the validation results. The algorithm is compared with the existing operational masks for SLSTR. The paper demonstrates how a neural net can be used to im- 
prove the performance over land significantly and proposes a new methodology for future cloud masking inter comparison activities.

\subsection{SLSTR}

The Sea and Land Surface Temperature Radiometer (SLSTR) instrument, Coppo et al. (2010), on board the Sentinel-3A and 3B satellite platforms is the latest in a series of dual view visible-infrared passive radiometers launched by ESA to measure sea ice, surface temperature, aerosols and clouds, Merchant et al. (2014); Ghent et al. (2017); Kokhanovsky and de Leeuw (2009); de Leeuw

70 et al. (2015); Poulsen et al. (2012). The record began in 1991 with (Along Track Scanning Radiometer) ATSR-1 on board (European Remote Sensing) ERS-1 followed by ATSR-2 (1995-2003) on board ERS-2 and (Advanced Along Track Scanning Radiometer) AATSR (2002-2012) on board Envisat. SLSTR on board Sentinel-3A was launched in 2016 followed shortly afterwards by Sentinel-3B in 2017. While the dual view which enables accurate atmospheric correction has been a consistent feature of all the instruments (although for SLSTR it is now a backward view rather than a forward view), the coverage and the number of channels has improved with each successive instrument. SLSTR now has a $1400 \mathrm{~km}$ Nadir view and a $740 \mathrm{~km}$ oblique view. When both satellites are operating, the revisit time at the equator is 0.8 days. Key channels from 2002 onwards are the $.55 .66 .87,1.63 .711$ and $12 \mu \mathrm{m}$ channels. For SLSTR, 1.3 and $2.2 \mu \mathrm{m}$ are new channels. The $1.3 \mu \mathrm{m}$ channel is a particularly useful addition for cloud identification as it is a water vapour absorption band particularly sensitive to cirrus clouds. The $2.2 \mu \mathrm{m}$ channel will be useful in this region, as in addition to the 1.6 channel it aids in the discrimination between snow and cloud (Schmit) et al. (2005)). The instrument specifications are shown in table 1. The instrument series is designed for high accuracy, with well calibrated measurements and low channel noise. Each instrument benefits from on board visible and infrared calibration as well as rigorous post launch vicarious calibration. 
Table 1: Characteristics of the SLSTR instrument. All channels are present in the nadir and oblique view. F1 and F2 indicate dedicated fire channels that are not used in this study.

\begin{tabular}{|l|c|c|c|}
\hline Channel name & Channel $\mu \mathrm{m}$ & Resolution $(\mathrm{km})$ & ATSR-2/AATSR \\
\hline S1 & .55 & 0.5 & yes \\
S2 & .66 & 0.5 & yes \\
S3 & .87 & 0.5 & yes \\
S4 & 1.37 & 1.0 & no \\
S5 & 1.61 & 1.0 & yes \\
S6 & 2.25 & 1.0 & no \\
S7 & 3.74 & 1.0 & yes \\
S8 & 10.85 & 1.0 & yes \\
S9 & 12.02 & 1.0 & yes \\
F1 & 3.74 & 1.0 & no \\
F2 & 10.85 & 1.0 & no \\
\hline
\end{tabular}

90

\subsection{Review of ATSR and SLSTR cloud identification}

Current cloud identification techniques can be broadly classified as empir$i c a l$, with the mask based primarily (but not necessarily exclusively) on multi spectral thresholds. The advantage of this approach is its simplicity and speed to implement and the ability to switch on and off tests according to the applicacloud detection scheme calculates a probability of a clear sky for a given pixel using the observations from the satellite, with prior information about the atmosphere and surface conditions and the uncertainties in these variables Karlsson et al. (2015); Heidinger et al. (2012); Merchant et al. (2005)). The key advantage of this scheme is a probability quality variable and the use of a priori information to constrain the result. A third technique which has recently been applied are machine learning models as demonstrated by Jeppesen et al. (2019) and Sus et al. (2018). These algorithms are gaining momentum as they have demonstrated good performance; however they require large training data sets 
the detection of cloud from the ATSR series of instruments as part of the ESA Cloud_cci project. The ATSR cloud identification model was developed using AVHRR data as a proxy and the algorithm was transferred using coefficients. The advantage of this approach was that collocations between AVHRR and The major disadvantage of this approach was that it could not use the full information content of the ATSR instrument. Even so, the algorithm delivered good results in independent validation analysis described in Bulgin et al. (2018); Poulsen et al. (2019).

The existing operational SLSTR product provides a number of cloud masks which are briefly described below. The cloud masks will be evaluated in this paper together with the NN mask.

- Empirical (applicable over all surfaces) The Empirical cloud mask employs a series of tests aimed at identifying cloud over different surface types. Details of the tests are outlined in the ESA technical reporting, ESA (2019a). To summarise, the tests use thresholds on the visible and infrared channels as well as some spatial coherence tests. The cloud masking scheme is based on those employed for ATSR-2 and AATSR described in Závody et al. (2000) with additional tests developed specifically using the 2.2 and $1.3 \mu \mathrm{m}$ channels. Some of the tests depend on results from previous tests, hence it is important to consider the order they are applied.

- Bayesian/probabilistic (available over land) The operational Land probabilistic cloud mask scheme is independent of the visible channels. It compares simulated brightness temperatures and climatological information to detect if a scene is clear or not. Ghent et al. (2017)

- Bayesian/probabilistic (available over sea) The Bayesian cloud mask over sea uses apriori information from ECMWF numerical weather prediction fields and radiative transfer calculations to estimate the probability of a 
scene being cloud or clear. It estimates a probability of cloud from 0 to 1 and implements a threshold value of 0.1. A number of problems have been identified with the flag including over and under flagging of low stratus and fog and misflagging in coastal regions. ESA (2019b), Merchant et al. (2005).

A number of additional cloud masks have been developed for ATSR-2, ATSR and SLSTR, specifically over ice surfaces. Istomina et al. (2010) uses multi spectral look up tables while Jafariserajehlou et al. (2019) uses temporal information to identify cloud scenes.

\subsubsection{Issues with current cloud identification}

Current cloud masking for operational satellites are far from perfect for all applications and a number of key issues, outlined below, remain to be addressed.

- Conservative cloud masking: To avoid biases in surface temperature and aerosol retrievals, existing retrieval schemes usually adopt a conservative approach to cloud screening in order to avoid potential biasing effects. Bulgin et al. (2014). This means that the algorithm uses a cloud mask that has a small false positive rate which has both advantages and disadvantages: the advantage of this approach is that the retrievals are rarely biased locally by unidentified clouds that will make a surface temperature retrieval generally appear colder than it actually is or an aerosol optical depth higher; the disadvantage is that the global coverage of retrievals is significantly reduced and in areas of persistent or difficult to identify cloud coverage, there may be few, if any, surface or atmospheric measurements. In Holzer-Popp et al. (2013), cloud masking was identified as one of the key reasons for the differences in satellite aerosol retrieval performance.

- One size fits all: Cloud masks, particularly operational ones, are often designed with a single use case in mind with the underlying assumption being that a single cloud mask is suitable for all users. Often only a binary 
cloud mask is provided. In some cases, thick aerosol plumes are masked out as it may impact the surface retrieval. However, this will bias a global large set of input information provides a number of weak identifiers for the identification. The algorithm developed here is strictly based on the information in a given pixel and doesn't make use of any information from neighbouring pixels or the complete SLSTR image. The overall approach to developing the aerosol retrieval.

- Uncertainty definitions can be confusing: While uncertainty measures are proving an invaluable product for satellite retrieval products such as surface temperature; how this uncertainty is applied to cloud identification is often unclear. For example: is the uncertainty a measure of the likelihood of a cloud or not, or is it a measure of the impact on the geophysical property the user is trying to retrieve. A case in point is that a warm low optically thin cloud could have a high uncertainty because it is difficult to identify but have little impact on the retrieved surface variable.

\section{Neural net based algorithm}

The use of a NN for cloud identification is motivated by the fact that a algorithm can be summarised as:

- Create a dataset that has truth labels attached to the SLSTR pixels. This truth label tells the algorithm, during training and validation, whether the pixel in question is cloudy or not.

- Split the dataset into two. One is used for training the algorithm and one is used for validation and provides feedback to the algorithm on for how long the training should continue.

- Train the NN to predict the truth labels of the validation sample in the best possible way through adjusting its internal parameters.

- Save the NN configuration such that it can be used on independent data where no truth labels are present. 


\subsection{Review of datasets for training and evaluation}

Any machine learning algorithm that depends on supervised learning for its configuration will rely critically on the creation of the dataset that it is trained on.

In the past ATSR and SLSTR cloud masks have been trained and evaluated using hand classified scenes. Bulgin et al. (2014). While each hand classified scene has many thousands of pixels, and is in general useful for training and evaluation, there are a number of disadvantages of this approach:

- Hand classified images are expensive to produce and as a consequence very few hand classified images are made publicly available. The delay time between creation and release time can be very long, stifling innovation.

- Because there are relatively few hand classified images, they cover only a few select regions, and the cloud types within the images will be highly correlated, reducing significantly the global representivity.

- The hand classification can be quite subjective for difficult to classify clouds, particularly for cloud edges, clouds that have small optical depth, and clouds over bright surfaces such as desert or ice.

- There is little added extra information in the mask to enable a more insightful cloud mask to be produced, e.g cloud type, height or optical thickness.

An alternative approach has been to consider instruments such as MODIS or AVHRR as a proxy as in Hollstein et al. (2015) and Sus et al. (2018). Such an approach doesn't take into account the different spectral shape of the individual channels, the associated noise, calibration, instrument geometry and the additional channels available in SLSTR.

口 In other cases, the mask is developed using surface synoptic observations (SYNOP) (Istomina et al. (2010)). However, these observations, while plentiful in time, are highly subjective and only located over land. 
In this paper we will use collocated CALIOP measurements to train and

\subsubsection{CALIOP}

Since 2006, the Cloud-Aerosol Lidar with Orthogonal Polarization (CALIOP) has been flown on board the CALIPSO satellite (Winker et al. (2009)). The instrument and active lidar utilise the two wavelengths $532 \mathrm{~nm}$ and $1064 \mathrm{~nm}$ is $333 \mathrm{~m}$ and the vertical resolution is $30-60 \mathrm{~m}$. In this analysis, we use the CAL_LID_L2 V4-20 $1 \mathrm{~km}$ and $5 \mathrm{~km}$ products. As statistical noise is averaged out, the sensitivity to cirrus cloud is higher for the $5 \mathrm{~km}$ than for the $1 \mathrm{~km}$ product; however, the $1 \mathrm{~km}$ product was used to train the $\mathrm{NN}$ as that resolu- 

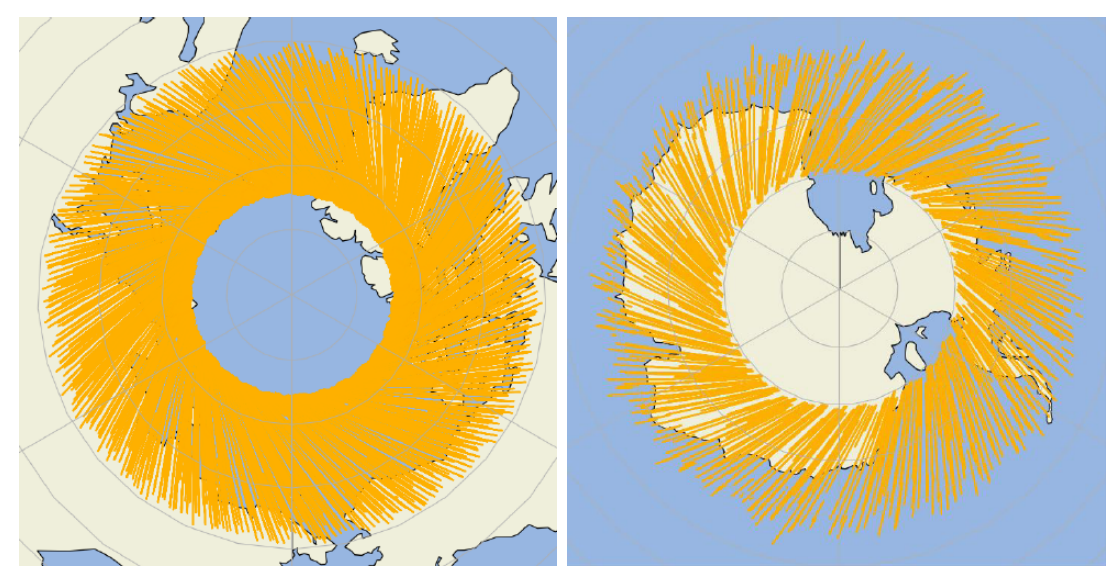

Figure 1: The distribution of the collocated pixels between SLSTR and CALIOP across the two polar regions. The lower number of matched pixels in the Antarctic region is due to the fact that that the time period used had less daytime than the Arctic region (and that only daytime matches are used).

As a result of the collocation, there are three million matched SLSTR pixels that have a nearly uniform distribution across the polar regions as illustrated in Fig.1. In the Arctic, collocated data is between $66.1^{\circ} \mathrm{N}$ and $81.0^{\circ} \mathrm{N}$, while Antarctic data is between $61.8^{\circ} \mathrm{S}$ and $78.1^{\circ} \mathrm{S}$. A common problem with datasets used for training neural nets is that each entry in the dataset is highly correlated to other entries. If that is the case, the dataset effectively behaves as a smaller dataset. If a low number of SLSTR images with all pixels matched are used for training that is exactly what will happen; they cover only small periods in time and have many pixels with the same clouds and surface types. As the matched dataset in our approach only cover a very thin strip across an SLSTR image, the overall dataset will only have small correlations between the pixels. Thus the dataset is more powerful to train on than a similar number of matched pixels in a low number of SLSTR images. The disadvantage is that collocations within a short time period are only possible in the polar regions. With a training dataset that covers a full year and has a very homogeneous coverage within the polar regions, it will cover all surface types as well as all lighting conditions.

In the SLSTR data, 22 inputs were identified to be used as features for the 
zenith angle, solar zenith angle, surface type flags and some ancillary information. The surface types Coastline, Ocean, Tidal, Dry Land, Inland water were provided as individual binary input channels rather than as a single bitmask. The Dry Land mask is obtained by taking the pixels labelled as Land but subtracting those labelled as Inland Water. The ancillary information Cosmetic, Duplicate, Day and Twilight were provided as binary input channels as well. The first two can inform the algorithm about how much the information can be trusted while the latter two, while fully correlated with the solar zenith angle allows for categorising afterwards. The Snow flag was not used as it contains information derived from other cloud identification algorithms and in addition was observed to be of low quality. Sun glint information was not used as it is never an issue in polar regions.

\subsection{Neural net}

The NN chosen was a simple feed forward net with the implementation based on TensorFlow (Abadi et al. (2015)). The geometry of the net was, using trial and error, developed as the one which produced optimal performance given the limited size of the test data. It has an input layer that consists of the 22 inputs outlined in the previous section. There are then four hidden layers where each neuron is connected to all neurons in the previous layer. Each hidden layer has layers were during training subject to dropout with a keep probability of 0.8 . Dropout is a technique that aims to prevent overfitting by randomly dropping neurons during training to prevent the net from being reliant on certain patterns. The output layer consists of two neurons using 'softmax' activation, producing outputs in the form $[\hat{y}, 1-\hat{y}]$. The net aims to produce an output of $[0,1]$ for a clear input pixel and $[1,0]$ for cloudy pixels. Although the output could be simplified to produce a single output $\hat{y}$, this two output approach allows the usage of 'Binary crossentropy' as a loss function. All the internal parameters of the neural net were optimised using the Adam optimiser, Kingma and Ba 
295

the training and validation samples in a random way with an 85:15 split. On a single core of an Intel(R) Xeon(R) CPU E5-2640 v3 without a dedicated GPU, the training took a few hours. After the model is trained, the weights are saved such that the model can be restored at a later point. The size of the neural net both in terms of the number of hidden layers and the number of neurons in each layer was examined. The performance improves when adding more layers and more neurons in each layer, but the further improvement after 4 hidden layers with 32 neurons in each was found to be marginal. However, with a larger collocated dataset that could be obtianed in the future, it is likely that a larger net would be able to further improve the performance.

\section{Performance}

This section will start out with specific qualitative examples of the NN to label scenes from SLSTR in comparison to the Bayesian and Empirical masks. We will then define the specific metrics used for a detailed quantitative comparison that in the subsequent subsections are evaluated according to surface type, cloud type and optical depth.

For each pixel in an SLSTR image, the trained NN provides an output between 0 and 1 . An output close to 0 means that the algorithm is confident that the pixel is clear while an output close to 1 means that the algorithm is confident that the pixel is cloudy. The way that this information is usually used, is that a threshold is defined and everything with an output below this threshold is classified as clear and everything above it as cloudy. The choice of threshold depends on the use case. A very low threshold will be able to identify clear areas with very few cloudy pixels wrongly classified, while a high threshold 320 will identify cloudy areas with a very low contamination of clear pixels. The optimal threshold to use might depend on the surface type. 


\subsection{Classification examples}

For visual comparisons to other algorithms, we will focus on the ability to label areas as clear. In Fig.2 four images are shown of an area of the Kamchatka peninsula. The Bayesian and Empirical masks are illustrated as well as the output of the NN, and a comparison of the Bayesian mask and the NN treated as a binary mask with a threshold. The comparison illustrates how larger areas (the blue ones) over the ocean are identified as clear by the NN compared to the Bayesian mask. On the other hand, the red areas, predominantly over land, are areas that the Bayesian algorithm identifies as clear but the NN doesn't. Most likely this is due to the Bayesian algorithm wrongly identifying many cloudy areas over land as clear as will later be discussed in relation to Fig. 5 . It can be seen that the Empirical algorithm is labelling an even larger area of the scene as cloudy. A similar set of comparisons are made in Fig. 3 for a scene dominated by twilight over the Antarctic coast. In that scene the Empirical mask completely fails over the ice sheet and has a noticeable boundary effect along the coastline.

\subsection{Metrics}

For a given algorithm, with a specific choice of threshold on the classifier if relevant, the True Positive Rate (TPR) and the False Positive Rate (FPR) for a sample of truth labelled data can be defined as

$$
\begin{aligned}
\mathrm{TPR} & =\frac{\# \text { pixels correctly identified as cloudy }}{\# \text { cloudy pixels }} \\
\mathrm{FPR} & =\frac{\# \text { pixels wrongly identified as cloudy }}{\# \text { non-cloudy pixels }} .
\end{aligned}
$$

The perfect algorithm would have $\mathrm{TPR}=1$ and $\mathrm{FPR}=0$. For an algorithm with an adjustable threshold, it is possible to draw a curve, called the Receiver Operating Characteristic (ROC) curve, of TPR as a function of FPR as seen in Fig. 4. A threshold of zero will correspond to everything being labelled as cloudy, and thus have $(\mathrm{FPR}, \mathrm{TPR})=(1,1)$ while a threshold of 1 will label everything as clear and have $(\mathrm{FPR}, \mathrm{TPR})=(0,0)$. A random classifier would 
(a)
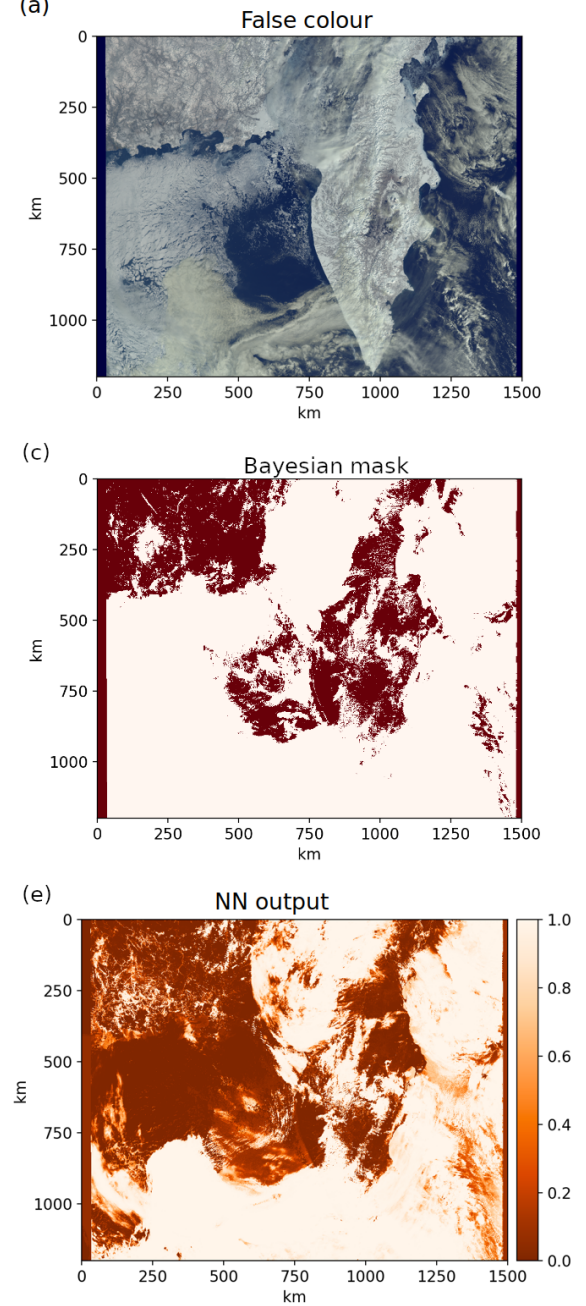

(b)

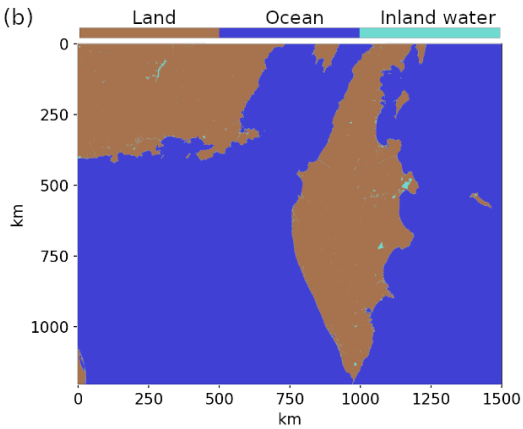

(d)

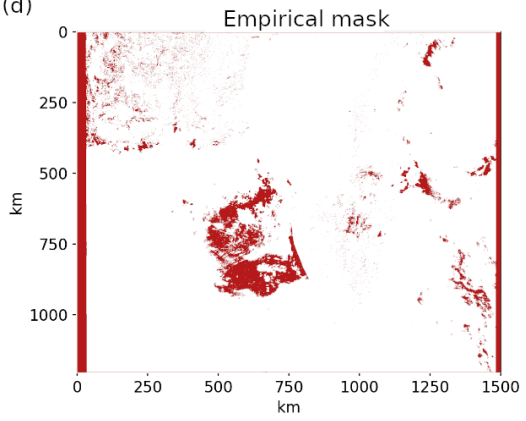

(f) Red: Bayesian, White: both, Blue: NN

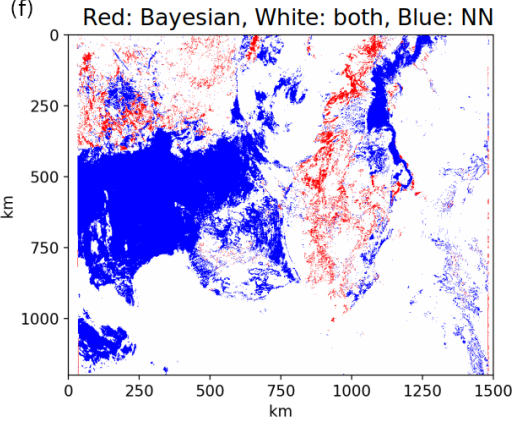

Figure 2: SLSTR scene taken on the 25th March 2019 at 00:11:34 UTC over the Kamchatka peninsula. In the image there is coastline, tidal regions, sea ice, and snow. a) False colour image, b) surface types, c) Bayesian cloud mask (white cloudy, red clear), d) Empirical mask (white cloudy, read clear) e) Output from NN f) Difference between Bayesian and NN masks when a threshold of 0.5 was put on the NN output. White is where the algorithms agree, blue is where the NN labels it as clear but the Bayesian as cloudy, red is where the NN labels it as cloudy but the Bayesian as clear. 

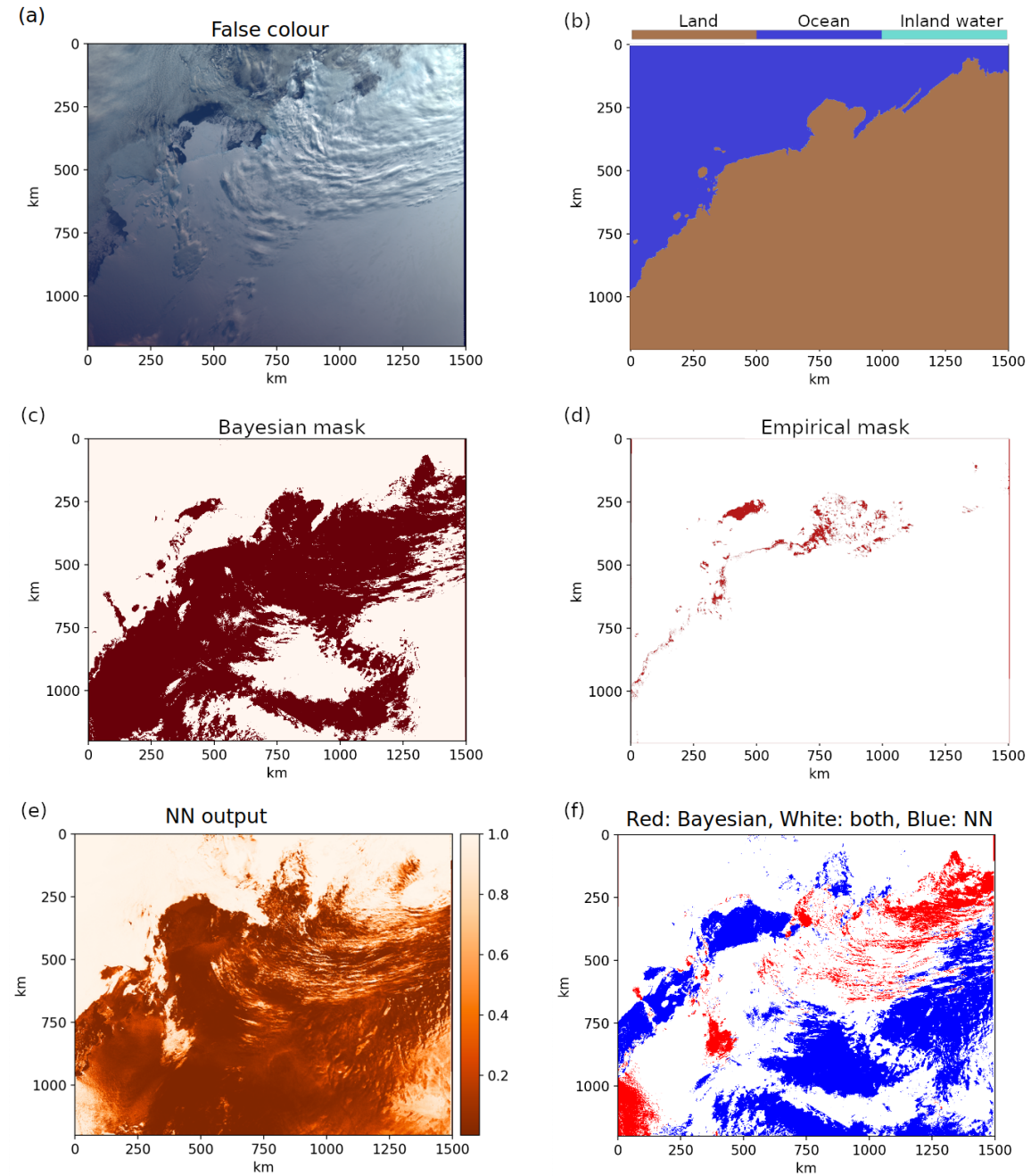

Figure 3: SLSTR scene taken on the 25th March 2019 at 00:47:34 UTC over the Antarctic coast. The images are of the same type as in Fig. 2 A large section of this image is classified as twilight. 


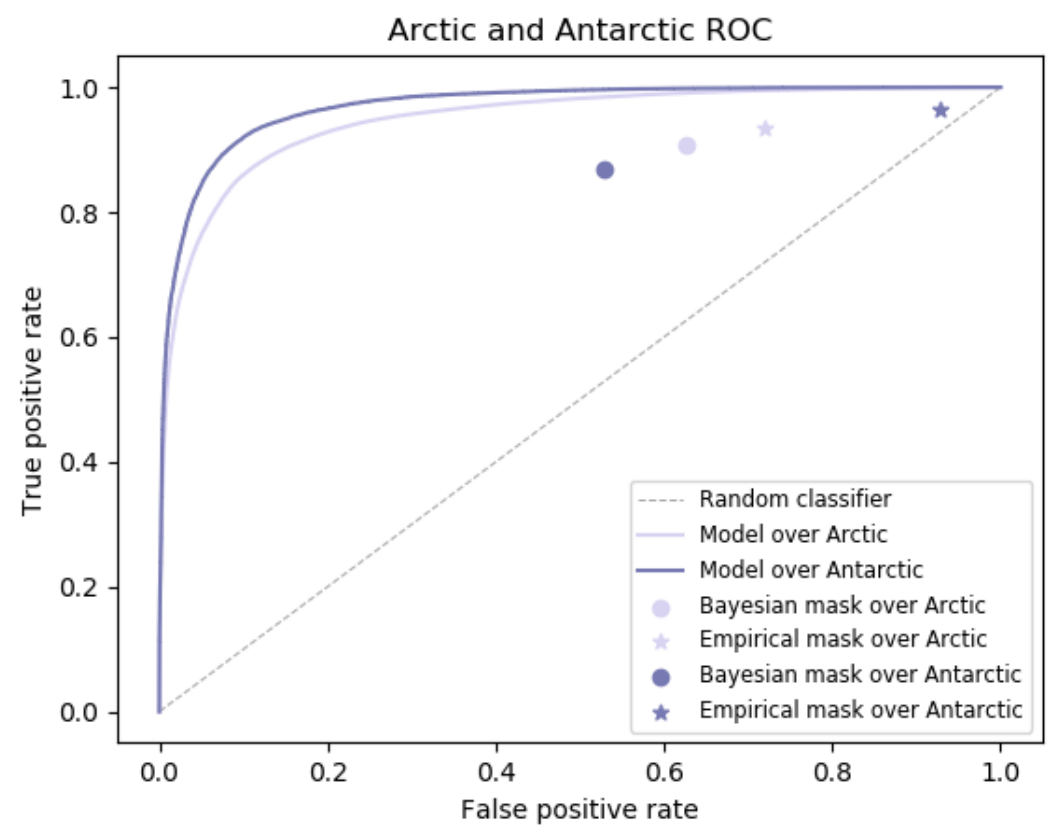

Figure 4: ROC curve for the neural net, Bayesian, and Empirical masks. Data has been separated into an Arctic and Antarctic data set for improved comparison. Also indicated on the graph is the expected behaviour of a random classifier.

simply connect these two points by a straight line, while a well performing algorithm would have the curve approach the top left corner of the plot.

To provide a single number that gives the performance of an algorithm, the area under the ROC curve is an often used measure. For a random algorithm, the area will be 0.5 , while for the perfect algorithm, it will be 1.0. Another often used metric for an algorithm is the Hanssen-Kuiper Skill Score (KSS), defined as KSS $=$ TPR - FPR (Karlsson and Johansson (2013) $)$. On the diagram of TPR as a function of FPR, lines of constant KSS are parallel to the random classifier line. A random classifier will have $\mathrm{KSS}=0$ while the perfect classifier will have $\mathrm{KSS}=1$.

When producing the ROC curves for the neural net, it is not possible to plot a corresponding curve for the existing cloud masks as their outputs are binary, 
Table 2: Comparison of the NN, the Bayesian and the Empirical algorithms. For the NN, the area under the ROC curve (AUC) is given and the Hanssen-Kuiper Skill Score (KSS) is evaluated at the point of its maximum along the ROC curve for the given line in the table. To compare two different types of algorithms, it is often more instructive to look at the figures of the true positive rate as a function of the false positive rate.

\begin{tabular}{|l|r|rr|c|c|}
\hline & \multirow{2}{*}{ Quantity } & \multicolumn{2}{|c|}{ NN } & Bayesian & Empirical \\
\cline { 3 - 6 } & 421214 & 0.95 & 0.77 & 0.28 & 0.17 \\
\hline All & 349967 & 0.95 & 0.75 & 0.27 & 0.20 \\
\hline Arctic & 71247 & 0.97 & 0.84 & 0.31 & 0.05 \\
Antarctic & 265666 & 0.96 & 0.78 & 0.12 & 0.11 \\
\hline Ocean & 151930 & 0.94 & 0.74 & 0.33 & 0.14 \\
Dry land & 3618 & 0.95 & 0.77 & 0.16 & 0.09 \\
Inland water & 348012 & 0.96 & 0.78 & 0.29 & 0.18 \\
\hline Day & 73202 & 0.93 & 0.71 & 0.22 & 0.15 \\
\hline
\end{tabular}

and there is no threshold to vary. In Fig. 4, the existing algorithms are thus given as a point and not a curve. When the point is below the ROC curve, it indicates poorer performance.

\subsection{Surface type}

The performances of the $\mathrm{NN}$ and the existing Bayesian and Empirical masks are evaluated on data that was not used for the training or validation of the NN. To provide truth level information, this data was still taken from the collocated sample with CALIOP. In Table 2, the Area Under the ROC Curve (AUC) and the Hanssen-Kuiper Skill Score (KSS) are compared for different sub-selections of the data. The KSS reported for the NN is the highest one achievable when changing the threshold for the subsample in question. In Fig 5 , the ROC curves of the NN for pixels classified as Ocean and Dry Land are compared to the single performance points for the Empirical and the Bayesian algorithms.

From the figure, it can be seen that for a comparable FPR, the performance 


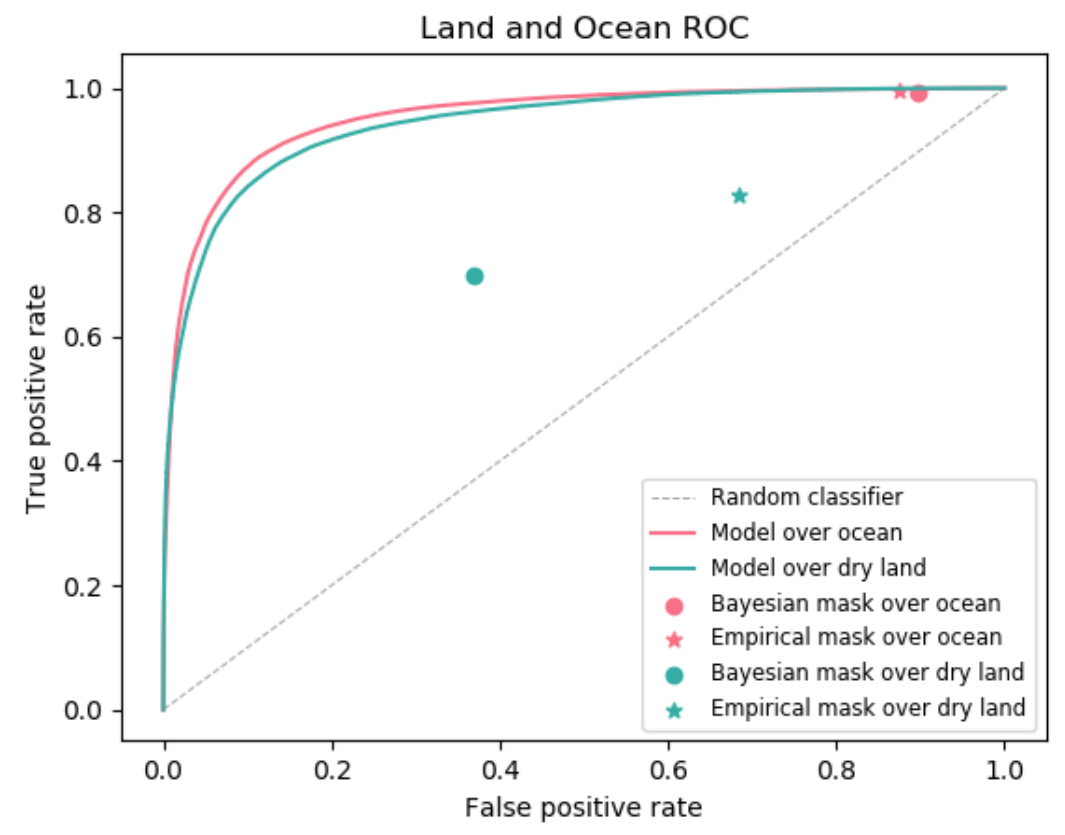

Figure 5: The ROC curves for the NN split pixels labelled as Ocean and Dry Land. The performance points of the Bayesian and the Empirical algorithms are marked as well. For the same False Positive Rate, it can be seen that all the algorithms have almost identical performance over Ocean while for Dry Land the NN performs much better. 
of all three algorithms are more or less the same over Ocean. However, the working point for the Empirical and Bayesian algorithms is very aggressive; if the aim is to identify clear areas, then only about $2 \%$ (1-TPR) of cloudy pixels will be labelled as clear, but the price is that only about $10 \%$ (1-FPR) of clear pixels are selected. For the NN, the same performance in terms of contamination of cloudy pixels in the sample of identified clear pixels can be achieved while retaining $40 \%$ of the clear pixels. This is what gives rise to the much higher KSS in the table. Thus the sample of clear pixels over Ocean can be made a factor 4 larger without compromising the quality of the data. As the Empirical and Bayesian algorithms only have a single working point it is impossible to say if those algorithms with relatively minor modification could lower the FPR while keeping the TPR high over Ocean.

For performance over Dry Land, the performance of the NN is in every measure much better than the Empirical and the Bayesian algorithms. From looking at Table 2, it can be seen that the NN has a very similar performance over all surface types. For Inland Water, the Bayesian algorithm has a KSS of 0.16 which might at first sight appear as good a performance as over water. However, it is with (FPR, TPR) values of $(0.66,0.82)$ which means that any sample identified as clear by the algorithm will have a high level of contamination with pixels that are actually cloudy $(1-\mathrm{TPR}=18 \%$ of cloudy pixels over inland water will be identified as clear). The Empirical algorithm suffers from the same performance issues as the Bayesian algorithm, but in an even more pronounced way. The NN does not suffer from that problem as can be seen from that the AUC stays essentially the same for all surface types.

\subsection{Cloud types}

In a similar way to that the performance of the NN can be investigated as a function of surface type, the performance can be investigated as a function of cloud type. In this case the TPR is evaluated only on the pixels that are classified from CALIOP as a given cloud type while the FPR is evaluated on all pixels 
as the comparison to the Bayesian and Empirical algorithms are given in Fig. 6 . The figure illustrates that for opaque clouds, all the algorithms perform very well. Indeed the $\mathrm{NN}$ is almost perfect in the sense that the ROC curve nearly reaches the optimal top left corner where identification is perfect with no false positives. For clouds with a degree of transparency (as defined by CALIOP) the performance begins to drop. For low, broken up cumulus, it can be seen that all algorithms have a hard time. With the different footprints of the SLSTR pixels and the strips of CALIOP for the truth matching, the truth labelling for broken up clouds will be of reduced quality and thus affect the truth labels used in both the training and the subsequent testing. The 20 minutes difference between the CALIOP and SLSTR time might also affect the performance for smaller clouds disproportionately. Unfortunately the amount of pixels with this cloud type in the testing sample is too small that the performance as a function of allowed matching time can be investigated. Broken up clouds can also be warm and of sub pixel size and for this reason difficult for the SLSTR instrument to detect.

\subsection{Optical depth}

The $5 \mathrm{~km}$ product from CALIOP provides a measurement of the optical depth of thin clouds. With the NN algorithm trained it is possible to compare the output from the NN, as defined in Sec. 3, for collocated pixels in bins of varying optical depth as seen in Fig. 7. As the $5 \mathrm{~km}$ product is used, each NN output produced is mapped to the nearest available optical depth measurement which may be up to $2.5 \mathrm{~km}$ away. For each bin, a distribution of NN output is created, and the median is plotted. Asymmetric error bars covers $34 \%$ of the distribution in each direction from the median. The figure demonstrates that the $\mathrm{NN}$ is correlated with the optical depth in the region between 0 and 2 optical depths. At higher values of the optical depth, the algorithm saturates. It can also be seen that the algorithm does not provide much separation between clear scenes and an optical depth up to 0.5 (corresponding to the first two bins). 

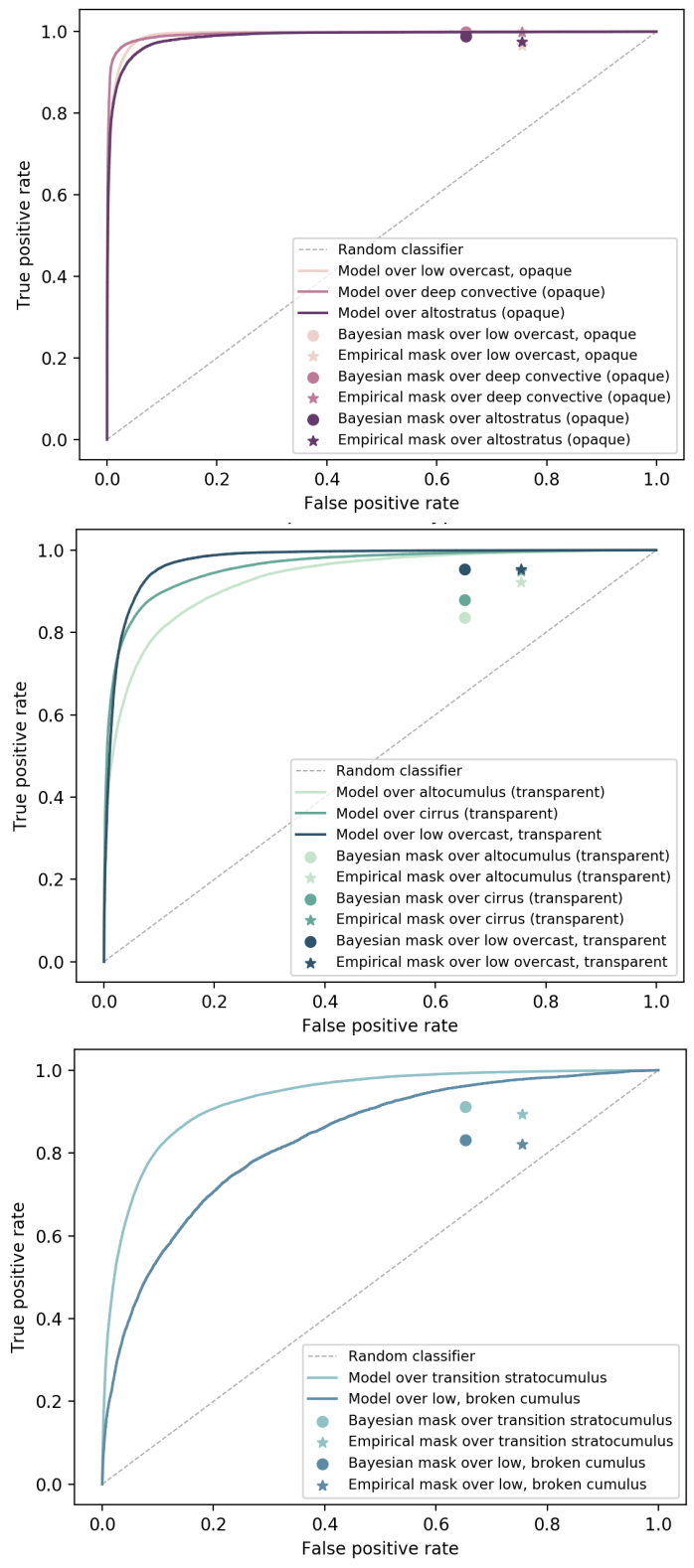

Figure 6: ROC curves for the NN algorithm divided into different types of clouds according to the CALIOP information. The performance of the Bayesian and the Empirical algorithms are overlaid as points. 


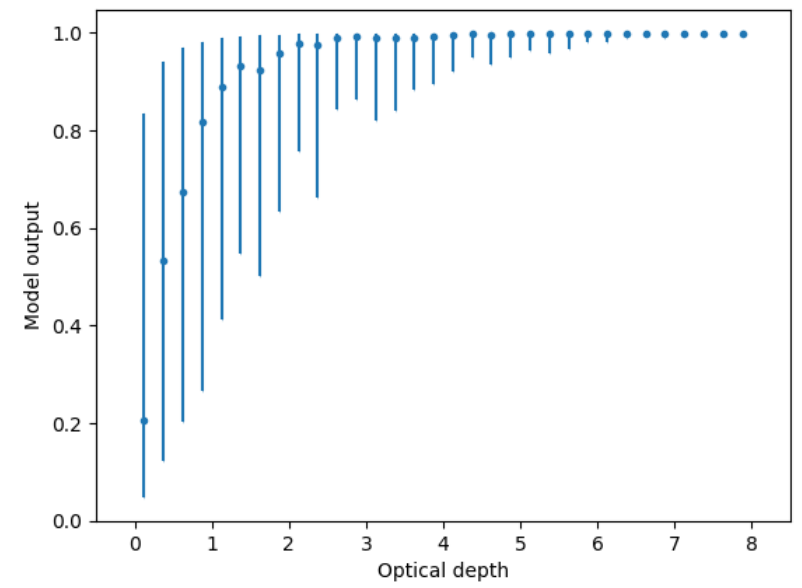

Figure 7: The output of the NN model, as defined in Sec. 3 binned according to the optical depth as measured in the $5 \mathrm{~km}$ product of CALIOP. In each bin, the data point represents the median of the distribution while the asymmetric error bars are reaching out from this to cover $34 \%$ in each direction. Each bin covers 0.25 in optical depth with the data point in the centre of the bin.

\subsection{Sensitivity of the neural net}

As the NN algorithm is far too complex to understand by simply inspecting its parameters, other methods have to be used to gain an understanding of which properties in the images are used for the identification of clouds. For this we use what is called a planing technique as presented in Chang et al. (2018). For a given variable, the planing algorithm will give the pixels in the training dataset weights such that for that specific variable there is no difference between the true cloud and true clear pixels as identified by CALIOP. The NN can then be trained with this weighted dataset and the performance evaluated again. The reduced performance will directly illustrate how sensitive the algorithm is to a given variable. The sensitivity to each of the input channels was measured in this way and the drop in performance measured by comparing the area under the ROC curve in each of the cases, with the result shown in Fig. 8. The planing method shows that the visible light channels individually are not so important for the 


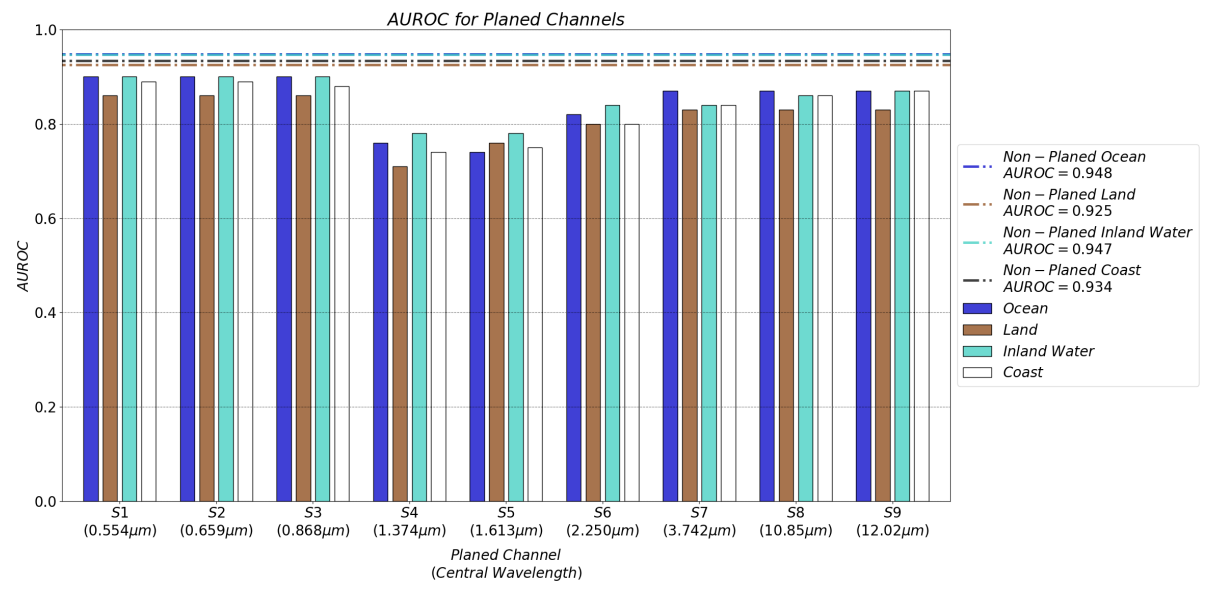

Figure 8: Sensitivity of the algorithm to each of the input channels. By removing an input channel from the training using a planing method, the area under the ROC curve (AUROC) is compared to the nominal performance shown as the dashed-dotted lines. It can be seen that excluding any channel leads to a drop in performance but with the largest drops related to $1.37 \mu \mathrm{m}$ and $1.61 \mu \mathrm{m}$ channels.

performance while the near infrared channels are. The loss in performance from planing a single variable is larger over land than over ocean. While the S5 and S6 channels are both water absorption channels, it can be seen that they add a large amount of different information to the cloud masking (if they were redundant, the performance of the NN would be unchanged by planing against one of them). They behave slightly differently according to the optical depth and effective radius of the cloud (Wang et al. (2018)). The channels also exhibit different spectral behaviour with respect to vegetation. The complex nature of these relationships serves to illustrate how a $\mathrm{NN}$ approach can provide added value through identification of the multi dimensional relationships between different channels, cloud types and the surface.

In a similar way we looked at the performance of the neural net when we made the training blind to the surface type and the longitude/latitude information. The result was that there was no impact on performance from removing this information within the statistical noise. For longitude and latitude, this is 
to be expected given the similarity of the polar regions across the range they were trained on. For the surface type, this is more surprising but matches up with that the algorithm has almost identical performance over land and sea. That surface type information is not important as truth information for the algorithm should not be taken to mean that the algorithm will perform well for surface types that it has not been trained on as will be seen through an example in the next section.

\section{Discussion}

Performance of the NN cloud identification scheme was analysed as a function of surface type and cloud type. This breakdown of the cloud mask performance enables a more comprehensive assessment of the cloud identification schemes and enables future cloud identification improvements to be more focused on the areas where current algorithms are underperforming.

The performance of the NN algorithm was slightly better over Antarctica than over the Arctic. The reason for this slight difference is not clear but could be because there are fewer fixed vegetation/snow sites in this polar region. The performance of this algorithm is significantly better than that described 475 in Poulsen et al. (2019) which achieved a KSS of 0.66 for the AATSR instrument in the polar regions compared with 0.77 for SLSTR. Compared with the algorithm applied to AATSR (but trained on AVHRR and transferred using coefficients) this algorithm was trained with SLSTR collocations with CALIOP. This algorithm includes the newest channels, 1.3 and $2.2 \mu \mathrm{m}$. The $1.3 \mu \mathrm{m}$ channel has shown to be of the highest importance to the cloud mask so will account for some but not all of this difference in skill.

Surprisingly the NN identification showed little difference in performance over land and sea with performance over sea only marginally better than over land. The operational masks on the other hand, which are in fact different applications of a Bayesian algorithm, showed quite different behaviour. The Empirical and Bayesian mask both performed well over sea while over land 
both performed poorly. The KSS scores for the Bayesian masks are consistently lower because the masks adopt a conservative approach to cloud identification.

The analysis showed that for opaque clouds, the operational and NN masks performed well. The divergence between the operational cloud mask and the $\mathrm{NN}$ increased when the clouds became more transparent and more broken. In these more difficult scenarios, while the NN showed a slight reduction in the performance, the NN cloud identification was significantly better than both the Empirical and Bayesian masks. This slight reduction in performance of the NN algorithm could be caused by the time difference between the SLSTR image and the CALIOP measurements used for the truth matching. When looking at pixels where the collocation with CALIOP has a time difference of less than 1 minute and comparing to when the time difference is between 19 and 20 minutes we see that the AOC drops by 0.07 . Unfortunately the amount of truth data does not allow to check if this minor drop in performance is specific to broken clouds.

The NN algorithm can be compared in a qualitative way to the algorithm presented in Jafariserajehlou et al. (2019). We do this by classifying the scene presented in Fig. 13 from that paper and showing it next to our classification in Fig. 9. It can be seen that in this example, the two algorithms provide very similar results. A quantitative comparison would require a project where both algorithms were compared using the identical scenes with the identical truth mapping.

In summary, the algorithm is significantly superior to the existing operational cloud masks in the polar regions where a direct quantitative comparison can be made.

While the algorithm was developed with training images over the polar regions, it is still possible to use it elsewhere. While there is no truth labelled dataset to check the performance against, a visual inspection reveals a very good performance over ocean but also some very poor performance over land. An example of this can be seen in Fig. 10 from a scene over northern Australia. It can be seen that the algorithm fails over the Australian red soil. This is not 

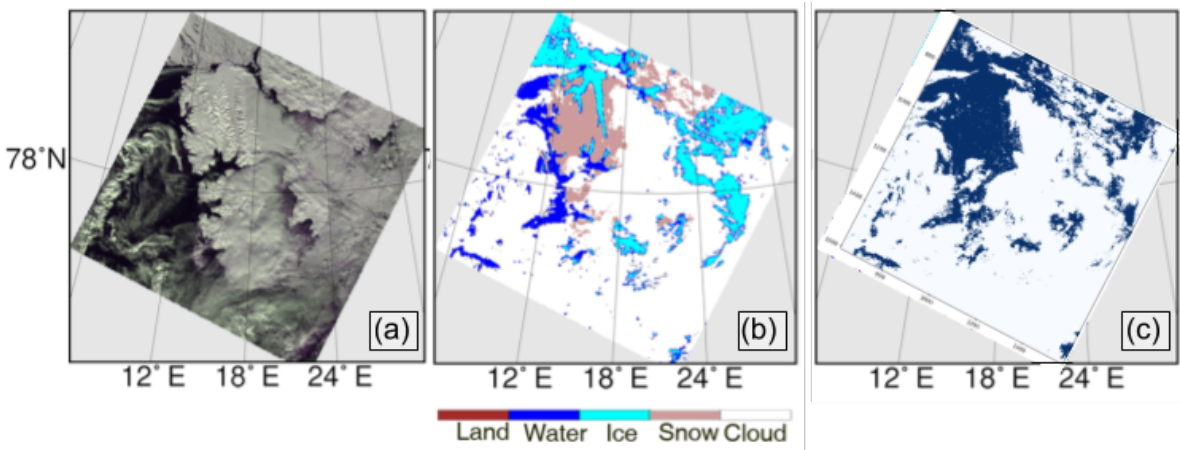

Figure 9: An SLSTR scene over Svalbard, taken at on 18 April 2017, 10:15:06 UTC. In (a) a false colour image of the scene, in (b) the classification as obtained with the algorithm presented in Jafariserajehlou et al. (2019) and in (c) the NN algorithm from this paper. A qualitative comparison shows that the two algorithms behave in a very similar way for this scene. Part (a) and (b) of this figure are taken from Jafariserajehlou et al. (2019).

surprising as this type of surface is very different to anything that the algorithm was trained on. In order to create a similar algorithm outside polar regions, where timely collocations over different surfaces (with a good representation of difference characteristics such as reflectance and temperature) are possible, truth data sets will need to defined. NASA's Cloud-Aerosol Transport System (CATS) lidar, Yorks et al. (2016), which operated between January 2015 and October 2017 has a small over lap period with SLSTR but the data was not available at the time of analysis. Hand classified data sets will be useful for this purpose, however these are generally time consuming and expensive to generate and will be subject to human biases and internally correlated.

The algorithm has not used the SLSTR backward view, in order to keep the developed mask consistent and applicable to the full swath. However, if the dual view swath is used, the information in the backward view could be exploited to improve the mask result. The algorithm could be developed further through using neighbouring pixels and with the addition of auxiliary data sets. 

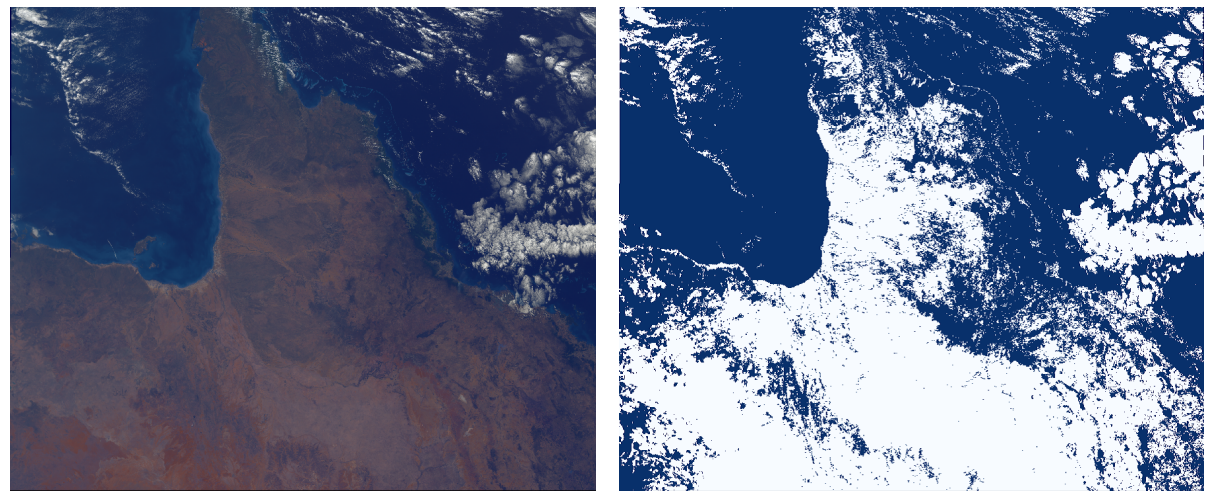

Figure 10: A false colour image over northern Australia with the corresponding mask from the NN with a threshold of 0.5 . It can be seen how the algorithm fails over a land type that it was not trained on while it still performs well over the ocean.

\section{Conclusion}

In this paper we have described the development of a machine learning algorithm to identify clear and cloudy pixels in SLSTR images. The algorithm was trained on collocated data from the CALIOP instrument. Collocations with good temporal matches between CALIOP and SLSTR are only possible in the polar regions which means that the power of using this method could only be shown there. We demonstrate a significantly improved performance compared to existing operational algorithms particularly over land. The algorithm performed equally well over land and sea. Opaque clouds were identified with greater skill than thin clouds and broken clouds. The sensitivity to optical depth was assessed using the CALIOP $5 \mathrm{~km}$ product which demonstrated that the ability to detect clouds dropped rapidly below 0.5 optical depths.

For the NN, the same performance in terms of contamination of cloudy pixels in the sample of identified clear pixels can be achieved while retaining $40 \%$ of the clear pixels compared to around 10\% with the existing Bayesian and Empirical cloud masks in the operational SLSTR product. Thus the sample of clear pixels over land and ocean can be made a factor 4 larger without paying a price in terms of quality of the data. This could have a significant impact on global 
values if there are regions of the globe that are systematically unrepresented. The algorithm is fast, just a few seconds per scene, so can be run operationally.

There is potential to improve the algorithm through the use of the oblique view as well and adding other auxiliary data sets. Improvements could also be made through using the texture in the image from a small region around the pixel that should be classified.

The methodology of using an active instrument such as CALIOP to provide truth information for the training of a $\mathrm{NN}$ to provide cloud masking from a passive instruments can no doubt be extended to other pairs of active and passive instruments, as long as a large collocated dataset can be provided.

Furthermore we have demonstrated a statistically significant validation method, with few inherent biases for evaluating cloud masks over what is a difficult region to discriminate cloud, even by eye, but a very important region with respect to understanding the climate.

\section{Author contribution statement}

Ulrik Egede and Caroline Poulsen: Conceptualization, Methodology, Formal analysis, Investigation, Resources, Writing - Original Draft, Writing Review \& Editing, Supervision, Project administration, Funding acquisition. Daniel Robbins, Barnaby Sandeford, Kenza Tazi, Thomas Zhu: Software, Investigation, Data curation, Formal analysis, Writing - Original Draft, Visualization.

\section{Acknowledgements}

The authors would like to thank Ed Polehampton and Caroline Cox at STFC RALSpace for help understanding the SLSTR cloud mask and Helen Brindley 575 for additional insights. For Caroline Poulsen, this work was partly undertaken in the Cloud_cci project (contract No.: 4000109870/13/INB) as part of the European Space Agency (ESA) CCI program. Ulrik Egede would like to acknowledge the support of STFC via grant number ST/N000242/1. 


\section{References}

${ }_{580}$ Abadi, M., Agarwal, A., Barham, P., Brevdo, E., Chen, Z., Citro, C., Corrado, G.S., Davis, A., Dean, J., Devin, M., Ghemawat, S., Goodfellow, I., Harp, A., Irving, G., Isard, M., Jia, Y., Jozefowicz, R., Kaiser, L., Kudlur, M., Levenberg, J., Mané, D., Monga, R., Moore, S., Murray, D., Olah, C., Schuster, M., Shlens, J., Steiner, B., Sutskever, I., Talwar, K., Tucker, P., Vanhoucke, V., Vasudevan, V., Viégas, F., Vinyals, O., Warden, P., Wattenberg, M., Wicke, M., Yu, Y., Zheng, X., 2015. TensorFlow: Large-scale machine learning on heterogeneous systems. URL: https://www.tensorflow.org/.

Bulgin, C., Merchant, C., Ghent, D., Klüser, L., Popp, T., Poulsen, C., Sogacheva, L., 2018. Quantifying uncertainty in satellite-retrieved land surface temperature from cloud detection errors. Remote Sensing 10, 616. doi:10.3390/rs10040616.

Bulgin, C., Sembhi, H., Ghent, D., Remedios, J., Merchant, C., 2014. Cloudclearing techniques over land for land-surface temperature retrieval from the Advanced Along-Track Scanning Radiometer. International Journal of Remote Sensing 35, 3594. doi $10.1080 / 01431161.2014 .907941$.

Chang, S., Cohen, T., Ostdiek, B., 2018. What is the machine learning? Physical Review D 97, 2. doi:10.1103/physrevd.97.056009.

Coppo, P., Ricciarelli, B., Brandani, F., Delderfield, J., Ferlet, M., Mutlow, C., Munro, G., Nightingale, T., Smith, D., Bianchi, S., Nicol, P., Kirschstein, S., Hennig, T., Engel, W., Frerick, J., Nieke, J., 2010. SLSTR: a high accuracy dual scan temperature radiometer for sea and land surface monitoring from

口 space. Journal of Modern Optics 57, 1815. doi 10.1080/09500340.2010. 503010.

ESA, 2019a. Cloud identification. URL: https://sentinels.copernicus.

605 a eu/web/sentinel/technical-guides/sentinel-3-slstr/level-1/ cloud-identification. 
ESA, 2019b. Slstr cloud identification product ntice. URL:

\https://sentinel.esa.int/documents/247904/3590270/ Sentinel-3A-SLSTR-Product-Notice-Level-1B-Version1.1.pdf.

Goosse, H., Kay, J.E., Armour, K.C., Bodas-Salcedo, A., Chepfer, H., Docquier, D., Jonko, A., Kushner, P.J., Lecomte, O., Massonnet, F., Park, H.S., Pithan, F., Svensson, G., Vancoppenolle, M., 2018. Quantifying climate feedbacks in

¿ polar regions. Nature Communications 9, 1919. URL: https://doi.org/10. 1038/s41467-018-04173-0, doi:10.1038/s41467-018-04173-0.

Heidinger, A.K., Evan, A.T., Foster, M.J., Walther, A., 2012. A naive bayesian cloud-detection scheme derived from CALIPSO and applied within PATMOS-

घ x. Journal of Applied Meteorology and Climatology 51, 1129. doi 10.1175/ JAMC-D-11-02.1.

Hollstein, A., Fischer, J., Carbajal Henken, C., Preusker, R., 2015. Bayesian cloud detection for MERIS, AATSR, and their combination. Atmospheric Measurement Techniques 8, 1757. doi:10.5194/amt-8-1757-2015.

Holzer-Popp, T., de Leeuw, G., Griesfeller, J., Martynenko, D., Klüser, L., Bevan, S., Davies, W., Ducos, F., Deuzé, J.L., Graigner, R.G., Heckel, A., von Hoyningen-Hüne, W., Kolmonen, P., Litvinov, P., North, P., Poulsen, C.A., Ramon, D., Siddans, R., Sogacheva, L., Tanre, D., Thomas, G.E., Vountas, M., Descloitres, J., Griesfeller, J., Kinne, S., Schulz, M., Pinnock, S., 2013. Aerosol retrieval experiments in the ESA Aerosol cci project. Atmospheric Measurement Techniques 6, 1919. doi 10.5194/amt-6-1919-2013.

Huang, Y., Dong, X., Bailey, D.A., Holland, M.M., Xi, B., DuVivier, A.K., Kay, J.E., Landrum, L.L., Deng, Y., 2019. Thicker clouds and accelerated Arctic 
sea ice decline: The atmosphere-sea ice interactions in Spring. Geophysical Research Letters 46, 6980. doi 10.1029/2019GL082791.

Istomina, L.G., von Hoyningen-Huene, W., Kokhanovsky, A.A., Burrows, J.P., 2010. The detection of cloud-free snow-covered areas using AATSR measurements. Atmospheric Measurement Techniques 3, 1005. doi 10.5194/ amt-3-1005-2010

Jafariserajehlou, S., Mei, L., Vountas, M., Rozanov, V., Burrows, J.P., Hollmann, R., 2019. A cloud identification algorithm over the Arctic for use with AATSR-SLSTR measurements. Atmospheric Measurement Techniques 12, 1059. doi $10.5194 /$ amt-12-1059-2019.

${ }_{645}$ Jeppesen, J.H., Jacobsen, R.H., Inceoglu, F., Toftegaard, T.S., 2019. A cloud detection algorithm for satellite imagery based on deep learning. Remote Sensing of Environment 229, 247. doi:10.1016/j.rse.2019.03.039

Karlsson, K.G., Johansson, E., 2013. On the Optimal Method for Evaluating Cloud Products from Passive Satellite Imagery using CALIPSO-CALIOP Data: Example Investigating the CM SAF CLARA-A1 Dataset. Atmospheric 口 Measurement Techniques 6, 1271-1286. URL: https://doi.org/10.5194/ amt-6-1271-2013, doi $10.5194 /$ amt-6-1271-2013

Karlsson, K.G., Johansson, E., Devasthale, A., 2015. Advancing the uncertainty characterisation of cloud masking in passive satellite imagery: Probabilistic formulations for NOAA AVHRR data. Remote Sensing of Environment 158, 126. doi $10.1016 /$ j.rse.2014.10.028

Kingma, D.P., Ba, J., 2014. Adam: A Method for Stochastic Optimization. arXiv e-prints arXiv:1412.6980

Kokhanovsky, A.A., de Leeuw, G. (Eds.), 2009. Oxford-RAL Aerosol and Cloud ${ }_{660}$ (ORAC): aerosol retrievals from satellite radiometers. Springer-Praxis. book section 7. p. 193. 
de Leeuw, G., Holzer-Popp, T., Bevan, S., Davies, W.H., Descloitres, J., Grainger, R.G., Griesfeller, J., Heckel, A., Kinne, S., Klüser, L., Kolmonen, P., Litvinov, P., Martynenko, D., North, P., Ovigneur, B., Pascal, N., Poulsen, C., Ramon, D., Schulz, M., Siddans, R., Sogacheva, L., Tanré, D., Thomas, G.E., Virtanen, T.H., von Hoyningen Huene, W., Vountas, M., Pinnock, S., 2015. Evaluation of seven European aerosol optical depth retrieval algorithms for climate analysis. Remote Sensing of Environment 162, 295. doi:10.1016/j.rse.2013.04.023.

Merchant, C.J., Embury, O., Roberts-Jones, J., Fiedler, E., Bulgin, C.E., Corlett, G.K., Good, S., McLaren, A., Rayner, N., Morak-Bozzo, S., Donlon, C., 2014. Sea surface temperature datasets for climate applications from Phase 1 of the European Space Agency Climate Change Initiative (SST CCI). Geoscience Data Journal 1, 179. doi:10.1002/gdj3.20.

Merchant, C.J., Harris, A.R., Maturi, E., Maccallum, S., 2005. Probabilistic physically based cloud screening of satellite infrared imagery for operational sea surface temperature retrieval. Quarterly Journal of the Royal Meteorological Society 131, 2735. doi 10.1256/qj.05.15.

Obu, J., Westermann, S., Bartsch, A., Berdnikov, N., Christiansen, H.H., Dashtseren, A., Delaloye, R., Elberling, B., Etzelmüller, B., Kholodov, A., Khomutov, A., Kääb, A., Leibman, M.O., Lewkowicz, A.G., Panda, S.K., Romanovsky, V., Way, R.G., Westergaard-Nielsen, A., Wu, T., Yamkhin, J., Zou, D., 2019. Northern Hemisphere permafrost map based on TTOP modelling for 2000-2016 at $1 \mathrm{~km} 2$ scale. Earth-Science Reviews 193, 299. doi: $10.1016 / j$.earscirev.2019.04.023.

Parkinson, C.L., 2019. A 40-y record reveals gradual Antarctic sea ice increases followed by decreases at rates far exceeding the rates seen in the Arctic. 口 Proceedings of the National Academy of Sciences 116, 14414. doi 10.1073/ pnas. 1906556116

Pörtner, H.O., Roberts, D.C., Masson-Delmotte, V., Zhai, P., Tignor, M., 
Poloczanska, E., Mintenbeck, K., Nicolai, M., Okem, A., Petzold, J., Rama, B., Weyer, N. (Eds.), 2019. Summary for Policymakers. Cambridge University Press, Cambridge, United Kingdom and New York, NY, USA. book section SPM. p. 1.

Poulsen, C., Siddans, R., Thomas, G., Sayer, A., Grainger, R., Campmany, E., Dean, S., Arnold, C., 2012. Cloud retrievals from satellite data using optimal estimation: Evaluation and application to ATSR. Atmospheric Measurement Techniques 5, 1889. doi:10.5194/amt-5-1889-2012.

Poulsen, C. Stengel, M., Thomas, G., Carboni, E., Povey, A., Hollmann, R., Christensen, M., McGarragh, G., 2019. Cloud_cci AATSR/ATSR-2 dataset version 3: 17 year climatology of global cloud and radiation properties. Earth System Science Data Discussions 2019, 1. doi 10.5194/essd-2019-217

Schmit, T.J., Gunshor, M.M., Menzel, W.P., Gurka, J.J., Li, J., Bachmeier, A.S., 2005. Introducing the next-generation advanced baseline imager on goes-r. Bulletin of the American Meteorological Society 86, 1079-

п 1096. URL: https://doi.org/10.1175/BAMS-86-8-1079, doi 10.1175/ BAMS-86-8-1079, arXiv:https://doi.org/10.1175/BAMS-86-8-1079.

Stocker, T., Qin, D., Plattner, G.K., Tignor, M., Allen, S., Boschung, J., Nauels, A., Xia, Y., Bex, V., Midgley, P. (Eds.), 2013. Summary for Policymakers. Cambridge University Press, Cambridge, United Kingdom and New York, NY, USA. book section SPM. p. 1. URL: www.climatechange2013.org, doi:10.1017/CB09781107415324.004.

Sus, O., Stengel, M., Stapelberg, S., McGarragh, G., Poulsen, C., Povey, A.C., Schlundt, C., Thomas, G., Christensen, M., Proud, S., Jerg, M., Grainger, R., Hollmann, R., 2018. The Community Cloud retrieval for CLimate (CC4CL) - Part 1: A framework applied to multiple satellite imaging sensors. Atmospheric Measurement Techniques 11, 3373. doi 10.5194/amt-11-3373-2018. Wang, J., Liu, C., Min, M., Hu, X., Lu, Q., Husi, L., 2018. Effects and applica- 
tions of satellite radiometer $2.25-\mu \mathrm{m}$ channel on cloud property retrievals. IEEE Transactions on Geoscience and Remote Sensing 56, 5207-5216.

Winker, D.M., Vaughan, M.A., Omar, A., Hu, Y., Powell, K.A., Liu, Z., Hunt, W.H., Young, S.A., 2009. Overview of the CALIPSO Mission and CALIOP Data Processing Algorithms. Journal of Atmospheric and Oceanic Technology 26, 2310. doi $10.1175 / 2009$ JTECHA1281.1.

725 Yorks, J.E., McGill, M.J., Palm, S.P., Hlavka, D.L., Selmer, P.A., Nowottnick, E.P., Vaughan, M.A., Rodier, S.D., Hart, W.D., 2016. An overview of the CATS level 1 processing algorithms and data products. Geophysical Research Letters 43, 4632. doi 10.1002/2016GL068006.

Závody, A.M., Mutlow, C.T., Llewellyn-Jones, D.T., 2000. Cloud clearing over the ocean in the processing of data from the along-track scanning radiometer ם (ATSR). Journal of Atmospheric and Oceanic Technology 17, 595. doi 10. 1175/1520-0426(2000)017<0595:CCOTOI>2.0.CO;2. 